Pacific Journal of Mathematics

A REMARK ON THE ISOTOPY CLASSES OF
DIFFEOMORPHISMS OF LENS SPACES 


\title{
A REMARK ON THE ISOTOPY CLASSES OF DIFFEOMORPHISMS OF LENS SPACES
}

\author{
W. C. HSiang AND B. JAhren
}

Let $L$ be a lens space of dimension at least 5 and with fundamental group of odd order. In this paper we reduce the computation of $\pi_{0}(\operatorname{Diff} L)$ to algebraic $K$-theory and homotopy theory. The approach is via parametrized surgery theory, as developed by the first author and $R$. Sharpe.

I. Introduction and statement of the result. In this note, we shall follow an idea of Browder's [1] and use the braid of groups of [8] (cf. [2]) to describe $\pi_{0}$ (Diff $\left.L^{2 n+1}\right)(n \geq 2)$ where $L^{2 n+1}$ is a lens space with $\pi=\pi_{1}\left(L^{2 n+1}\right)=\mathbf{Z}_{l}$ a cyclic group of odd order.

Let $\mathscr{P}\left(L^{2 n+1}\right)=\operatorname{Diff}\left(L^{2 n+1} \times[0,1] ; L^{2 n+1} \times 0\right)$ be the pseudo-isotopy space of diffeomorphism of $L^{2 n+1}$. Turning upside down, we have an involution '-' on $\pi_{0} \mathscr{P}\left(L^{2 n+1}\right)$ [6]. The quotient group

$$
\pi_{0} \mathscr{P}\left(L^{2 n+1}\right) /\{x-\bar{x}\}
$$

can be described as a cobordism group $\pi_{0}\left(\Re\left(L^{2 n+1}\right)\right)$ as follows. An object $\alpha=(W(\alpha), f)$ is a diagram

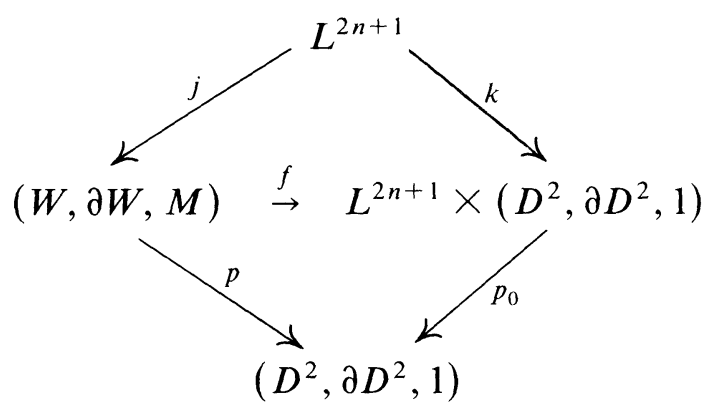

satisfying the following conditions:

(i) 1 is the base point of $\partial D^{2}$ and the upper triangle is commutative with $j$ a diffeomorphism and $k$ the standard identification of $L^{2 n+1}$ with $L^{2 n+1} \times 1$,

(ii) $f$ is a simple homotopy equivalence,

(iii) the lower triangle is commutative with $p_{0}$ the projection onto the second factor, and $L^{2 n+1} \stackrel{j}{\rightarrow} \partial W \stackrel{p}{\rightarrow} \partial D^{2}$ is a smooth fibration. 
Two such objects $\alpha_{1}, \alpha_{2}$ are cobordant if we have

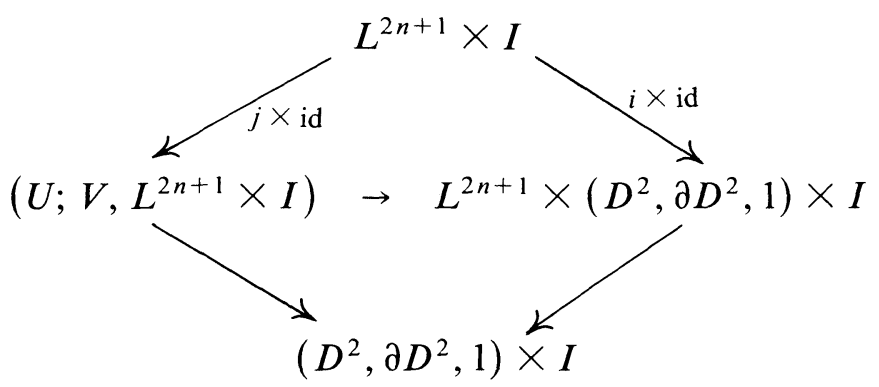

satisfying the following conditions:

(i) The upper triangle is commutative with $U$ an $s$-cobordism between $W\left(\alpha_{1}\right)$ and $W\left(\alpha_{2}\right)$,

(ii) the lower triangle is also commutative such that $L^{2 n+1} \rightarrow V \rightarrow$ $\partial D^{2} \times I$ is a smooth fibration,

(iii) $F \mid W\left(\alpha_{i}\right)(i=1,2)$ induces the object $\alpha_{i}(i=1,2)$.

There is a homomorphism

$$
r: L_{2 n+4}(\pi) \rightarrow \pi_{0} \Re\left(L^{2 n+1}\right)
$$

defined as follows. Let $x \in L_{2 n+4}(\pi)$ be represented by a normal cobordism

(3) $f:\left(U ; W, L^{2 n+1} \times D^{2}\right) \rightarrow L^{2 n+1} \times\left(D^{2} \times I ; D^{2} \times 1, D^{2} \times 0\right)$

such that $f \mid f^{-1}\left(L^{2 n+1} \times \partial D^{2} \times I\right)$ is diffeomorphism and $f \mid L^{2} \times D^{2}$ is the identity map. We can easily construct an object $\alpha=(W, f \mid W)$ representing $r(x)$ by restricting $f$ to $W$ and it is easy to verify that the cobordism $[\alpha] \in \pi_{0}\left(\Re\left(L^{2 n+1}\right)\right)$ is well-defined. In fact, $r$ may be viewed a homomorphism analogous to one of the maps in Rothenberg exact sequence [13] but we are now at the isotopy level. We will show that Coker $r$ is the group $\pi_{1}\left(C\left(L^{2 n+1}\right)\right)$ of the braid of groups of [8]. Recall that $\pi_{0}\left(\mathcal{C}\left(L^{2 n+1}\right)\right)$ also has a cobordism representation. (See [8, pp. 420-421].)

Let $S$ be the group of simple homotopy equivalences of $L^{2 n+1}$ to itself and let $H$ be the image of $\left[L^{2 n+1} \times I / L \times \partial I\right.$; Top/0] in $\left[L^{2 n+1} \times I / L \times \partial I ; G / 0\right]$.

We have the following result.

THEOREM 1.1. Let $L^{2 n+1}$ be a lens space with $\pi=\pi_{1}\left(L^{2 n+1}\right)=\mathbf{Z}_{l} a$ cyclic group of odd order. Then,

$$
\begin{aligned}
0 & \rightarrow \pi_{0}\left(\mathcal{C}\left(L^{2 n+1}\right)\right) \oplus H \rightarrow \pi_{0}\left(\text { Diff } L^{2 n+1}\right) \\
& \rightarrow S \rightarrow 0
\end{aligned}
$$


REMARKs. (1) For related work about real projective spaces, see $\mathrm{H}$. Schneider and R. Wells, A note on the concordance homotopy group of real projective space Proc. Amer. Math. Soc. 26 (1977), 367-373.

(2) It follows from [2], [7], [8] that $\pi_{0}\left(e\left(L^{2 n+1}\right)\right)$ is equal to $\mathrm{Wh}_{2}\left(\pi_{1} L^{2 n+1}\right) /\{x-\bar{x}\}$ mod 2-torsions. In general, $H$ is not easy to compute.

II. The braids of groups for $L^{2 n+1}$. Let Aut $L^{2 n+1}$ be the $H$-space of simple homotopy equivalences of $L^{2 n+1}$ to itself. We have the following fibration

$$
\mathscr{F}\left(L^{2 n+1}\right) \rightarrow \text { Diff } L^{2 n+1} \rightarrow \text { Aut } L^{2 n+1} .
$$

Then, a point in $\mathscr{F}\left(L^{2 n+1}\right)$ is represented by a pair $\left(\phi, \phi_{t}\right)$ where $\phi \in$ Diff $L^{2 n+1}$ and $\phi_{t}$ is a path in Aut $M$ connecting $\phi$ to Id $\in$ Diff $L^{2 n+1}$. Set

$$
L_{\phi}=L^{2 n+1} \times I /\{(m, 1) \sim(\phi(m), 0)\},
$$

the mapping torus of $\phi . \phi_{t}$ induces a simple homotopy equivalence

$$
F:\left(L_{\phi}, L^{2 n+1} \times 1\right) \rightarrow\left(L^{2 n+1} \times S^{1}, L^{2 n+1} \times 1\right) .
$$

Following [12], one can construct a space $\delta\left(L^{2 n+1} \times\left(S^{1}, 1\right)\right)$ of simple homotopy smoothings of $L^{2 n+1} \times S^{1}$ which are standard on $L^{2 n+1} \times 1$, and we have the map

$$
\tau: \mathscr{F}\left(L^{2 n+1}\right) \rightarrow \mathcal{S}\left(L^{2 n+1} \times\left(S^{1}, 1\right)\right)
$$

defined by $\tau\left(\left(\phi, \phi_{t}\right)\right)=F$. We also have a map

$$
\eta: S\left(L^{2 n+1} \times\left(S^{1}, 1\right)\right) \rightarrow G / 0^{\Sigma L^{+}}
$$

where $\Sigma L^{+}=L^{2 n+1} \times S^{1} / L^{2 n+1} \times 1$. Let us consider the following diagram of fibrations

$$
\begin{array}{ccccc}
\mathscr{B}\left(L^{2 n+1}\right) & = & \mathscr{B}\left(L^{2 n+1}\right) & & \\
\downarrow & & \downarrow & & \\
\mathcal{C}\left(L^{2 n+1}\right) & \rightarrow & \mathscr{F}\left(L^{2 n+1}\right) & \stackrel{\eta \cdot \tau}{\rightarrow} & G / 0^{\Sigma L^{+}} \\
\downarrow & & \downarrow & & \downarrow \\
\mathfrak{L}_{2}\left(L^{2 n+1}\right) & \rightarrow & \mathcal{S}\left(L^{2 n+1} \times\left(S^{1}, 1\right)\right) & \stackrel{\eta}{\rightarrow} & G / 0^{\Sigma L^{+}}
\end{array}
$$

where $\mathscr{B}\left(L^{2 n+1}\right), \mathcal{C}\left(L^{2 n+1}\right), \mathcal{L}_{2}\left(L^{2 n+1}\right)$ are the homotopy fibres of the obvious maps. It is easy to see that $\pi_{i}\left(\varrho_{2}\left(L^{2 n+1}\right)\right)=L_{2 n+\imath+3}(\pi)$ the 
surgery group of $\pi$. We have the following braid of groups of [8]:

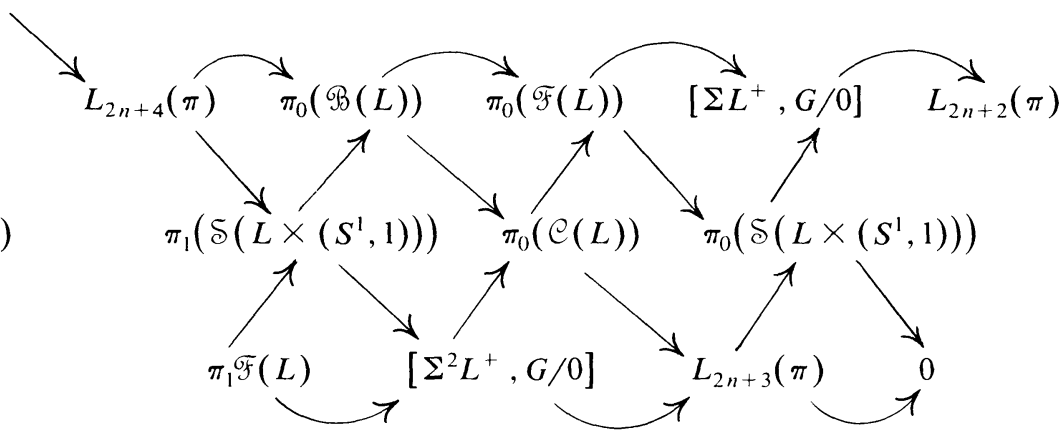

where $\Sigma^{2} L^{+}=L^{2 n+1} \times D^{2} / L^{2 n+1} \times S^{1}$ and $\pi_{0}\left(\delta\left(L^{2 n+1} \times\left(S^{1}, 1\right)\right)\right)$ is just the simple homotopy smoothings of $L^{2 n+1} \times S^{1}$ which is standard on $L^{2 n+1} \times 1$.

Replacing Diff $L^{2 n+1}$ by Homeo $L^{2 n+1}, \delta\left(L^{2 n+1} \times\left(S^{1}, 1\right)\right)$ by $\mathcal{S}^{\text {Top }}\left(L^{2 n+1} \times\left(S^{1}, 1\right)\right)$, we have a corresponding braid of groups

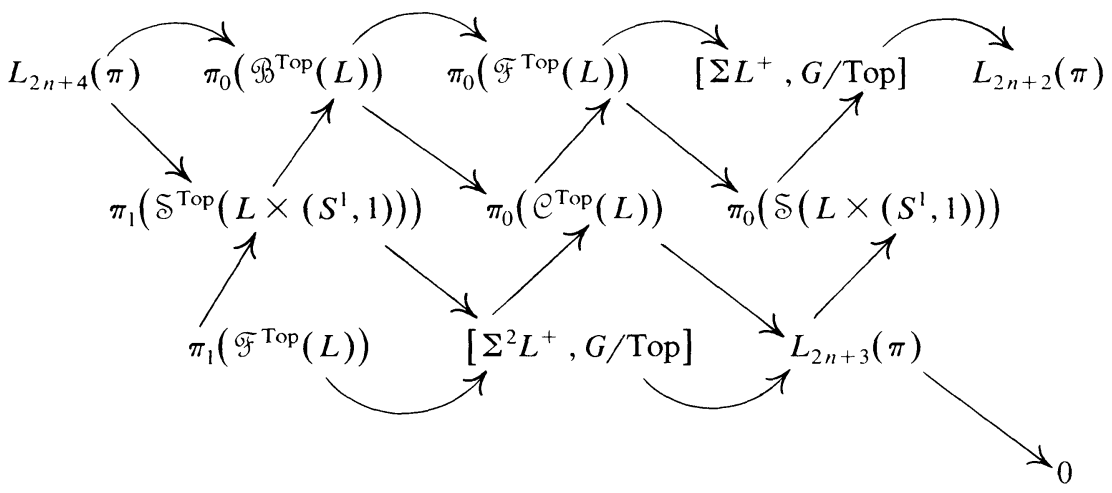

such that (6) is mapped into (7) in the obvious way. It follows from [2] and [6] that $\pi_{0}\left(\Re\left(L^{2 n+1}\right)\right) \cong \pi_{0}\left(\Re^{T o p}\left(L^{2 n+1}\right)\right)$ under the natural map of the above. Consequently, $\pi_{0}\left(\bigodot\left(L^{2 n+1}\right)\right)=\pi_{0}\left(\bigodot^{\text {Top }}\left(L^{2 n+1}\right)\right)$ again under the natural map of the above.

We shall also prove the following theorem.

THEOREM 2.1. Let $L^{2 n+1}(n \geq 2)$ be the lens space with $\pi=\pi_{1} L^{2 n+1}=$ $\mathbf{Z}_{l}$, a cyclic group of odd order. Then,

$$
0 \rightarrow \pi_{0}\left(\mathcal{C}\left(L^{2 n+1}\right)\right) \rightarrow \pi_{0}\left(\operatorname{Homeo}\left(L^{2 n+1}\right)\right) \rightarrow S \rightarrow 0 .
$$

REMARK. As we pointed out after Theorem 1.1,

$$
\pi_{0}\left(\bigodot\left(L^{2 n+1}\right)\right)=\mathrm{Wh}_{2}\left(\pi_{1} L^{2 n+1}\right) /\{x-\bar{x}\} \bmod 2 \text {-torsions. }
$$


III. Homotopy calculations. In this section we compute $\pi_{i}$ (Aut $L^{2 n+1}$ ) for $i=0,1$. Assume as before that $\pi_{1}\left(L^{2 n+1}\right)$ has odd order $l$.

Proposition 3.1. $\pi_{1}$ (Aut $L^{2 n+1}$ ) has order $2 l^{2}$, and the homomorphism $\pi_{1}\left(\right.$ Diff $\left.L^{2 n+1}\right) \rightarrow \pi_{1}\left(\right.$ Aut $\left.L^{2 n+1}\right)$ is epimorphic. Hence, $\pi_{1}\left(\right.$ Homeo $\left.L^{2 n+1}\right) \rightarrow$ $\pi_{1}$ (Aut $\left.L^{2 n+1}\right)$ is also epimorphic.

Proof. Let us consider the following map of fibrations.

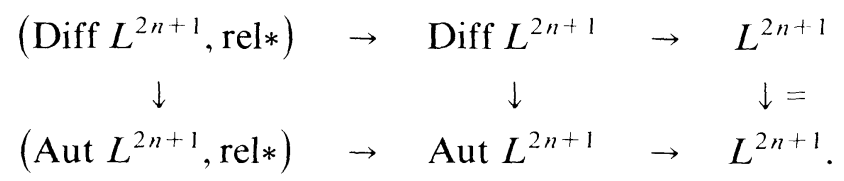

We have the following commutative diagram of $\pi_{1}$ :

$$
\begin{aligned}
& 0 \rightarrow \pi_{1}\left(\operatorname{Diff}\left(L^{2 n+1}, *\right)\right) \rightarrow \pi_{1}\left(\operatorname{Diff} L^{2 n+1}\right) \rightarrow \pi_{1}\left(L^{2 n+1}\right) \rightarrow 0 \\
& 0 \rightarrow \pi_{1}\left(\operatorname{Aut}\left(L^{2 n+1}, *\right)\right) \rightarrow \pi_{1}\left(\text { Aut } L^{2 n+1}\right) \rightarrow \pi_{1}\left(L^{2 n+1}\right) \rightarrow 0 .
\end{aligned}
$$

In order to prove the proposition, it suffices to show that

$$
\pi_{1}\left(\operatorname{Diff}\left(L^{2 n+1}, *\right)\right) \rightarrow \pi_{1}\left(\operatorname{Aut}\left(L^{2 n+1}, *\right)\right)
$$

is epimorphic and $\pi_{1}\left(\operatorname{Aut}\left(L^{2 n+1}, *\right)\right)$ is a cyclic group of order $2 l$.

Let $L^{2 n+1}=L^{2 n+1}\left(q_{0}, \ldots, q_{n}\right)$ be the quotient of $S^{2 n+1}$ by the subgroup $\mathbf{Z}_{l} \subset U(n+1)$ generated by

$$
\left(\begin{array}{ccc}
e^{2 \pi l q_{0} / l} & & \\
& \ddots & \\
& & e^{2 \pi i q_{n} / l}
\end{array}\right)
$$

Let $*=(1,0, \ldots, 0)$ be the base point and define $h: S^{1} \times L^{2 n+1} \rightarrow L^{2 n+1}$ by $\left(e^{i \theta} ; z_{0}, \ldots, z_{n}\right) \rightarrow\left(z_{1}, \ldots, z_{n} e^{i \theta}\right)$. We claim that the image of $[h] \in$ $\pi_{1}\left(\operatorname{Diff}\left(L^{2 n+1}, *\right)\right)$ in $\pi_{1}\left(\operatorname{Aut}\left(L^{2 n+1}, *\right)\right)$ generated a group $\mathbf{Z}_{2 /}$. For this purpose let us consider the following commutative diagram

$$
\begin{array}{ccc}
\pi_{1}\left(\operatorname{Diff}\left(L^{2 n+1}, *\right)\right) & \rightarrow & \pi_{1}\left(\operatorname{Diff}\left(S^{2 n+1}, *\right)\right) \\
\downarrow & & \downarrow \\
\pi_{1}\left(\operatorname{Aut}\left(L^{2 n+1}, *\right)\right) & \rightarrow & \pi_{1}\left(\operatorname{Aut}\left(S^{2 n+1}, *\right)\right)=\mathbf{Z}_{2}
\end{array}
$$


by lifting the maps to the universal cover of $L^{2 n+1}$. Since $l$ is odd, the composite

$$
\pi_{1}\left(\operatorname{Diff}\left(L^{2 n+1}, *\right)\right) \rightarrow \pi_{1}\left(\operatorname{Aut}\left(L^{2 n+1} ; *\right)\right) \rightarrow \pi_{1}\left(\operatorname{Aut}\left(S^{2 n+1}, *\right)\right)=\mathbf{Z}_{2}
$$

is epimorphic and the image of $[h]$ is a generator of

$$
\pi_{1} \operatorname{Aut}\left(S^{2 n+1}, *\right)=\mathbf{Z}_{2} \text {. }
$$

In order to complete our argument, we consider the first two stages of the Postnikov system of $L^{2 n+1}$

$$
\begin{array}{ccccc}
K\left(\mathbf{Z}_{2}, 2 n+2\right) & \stackrel{i_{2}}{\rightarrow} & E_{2} & & \\
& & \downarrow & & \\
K(\mathbf{Z}, 2 n+1) & \stackrel{l_{2}}{\rightarrow} & E_{1} & \stackrel{k_{2}}{\rightarrow} & K\left(\mathbf{Z}_{2}, 2 n+3\right) \\
& & & & \\
& & K\left(\mathbf{Z}_{l}, 1\right) & \stackrel{k_{1}}{\rightarrow} & K(\mathbf{Z}, 2 n+2) .
\end{array}
$$

The $k$-invariants are given as follows. $k_{1}^{*}\left(\tau_{2 n+2}\right)=\left(\beta\left(\tau_{1}\right)\right)^{n+1}$ where $\tau_{2 n+2}$ $\in H^{2 n+2}\left(\mathbf{Z}, 2 n+2 ; \mathbf{Z}_{2}\right), \tau_{1} \in H^{1}\left(\mathbf{Z}_{l}, 1 ; \mathbf{Z}_{l}\right)$ are the generators and $\beta: H^{1}\left(\mathbf{Z}_{l}, 1 ; \mathbf{Z}_{l}\right) \rightarrow H^{2}\left(\mathbf{Z}_{l}, 1 ; \mathbf{Z}\right)$ is the Bockstein homomorphism; $k_{2}^{*}\left(\tau_{2 n+3}\right)=\operatorname{Sq}^{2}\left(\left(\tau_{2 n+1}\right)_{2}\right)$ where $\tau_{2 n+3}$ is the fundamental generator of $H^{2 n+3}\left(\mathbf{Z} ; 2 n+3 ; \mathbf{Z}_{2}\right)$ and $\left(\tau_{2 n+1}\right)_{2} \in H^{2 n+1}\left(E_{2} ; \mathbf{Z}_{2}\right)$ is the $\bmod 2$ reduction of a class which pulls back to a fundamental class of $L^{2 n+1}$.

Given a map $f: S^{1} \times L^{2 n+1} \rightarrow K\left(\mathbf{Z}_{l}, 1\right)$ such that $k_{1} f \simeq 0$, $H^{2 n+1}\left(S^{1} \times L^{2 n+1} ; \mathbf{Z}\right)$ acts transitively on the set of all the lifting to $E_{1}$ and the stability subgroup of a lifting is the image of a certain homomorphism

$$
S \Delta\left(k_{1}, u\right): H^{0}\left(S^{1} \times L^{2 n+1} ; \mathbf{Z}_{2}\right) \rightarrow H^{2 n+1}\left(S^{1} \times L^{2 n+1} ; \mathbf{Z}\right) .
$$

(Cf. [14], pp. 448-452 for the notation $S \Delta\left(k_{1}, u\right)$.) Since $\left(k_{1} \circ f\right)^{*}=0$ $S \Delta\left(k_{1}, u\right)=0$ for all $u$. Recall that $\pi_{1}$ (Aut $L^{2 n+1}$ ) is the set of all homotopy classes of maps $S^{1} \times L^{2 n+1} \rightarrow L^{2 n+1}$ which restricts to the identity on $* \times L^{2 n+1}$, and this reduces the number of possible liftings to $l^{2}$, coming from the variations of the composite map $S^{1} \subset S^{1} \times L \rightarrow K\left(\mathbf{Z}_{l}, 1\right)$ and the torsion subgroup of $H^{2 n+1}\left(S^{1} \times L^{2 n+1} ; \mathbf{Z}\right)$.

On the other hand, it follows from the second Peterson-Stein formula [11] that all these liftings to $E_{1}$ can be further lifted to $E_{2}$. This time the set of the liftings is determined by an action of (a quotient of) $H^{2 n+2}\left(S^{1} \times L^{2 n+1} ; \mathbf{Z}_{2}\right)=\mathbf{Z}_{2}$. In fact, this action can be geometrically realized as follows. 
Given $g: S^{1} \times L^{2 n+1} \rightarrow L^{2 n+1}$ and $0 \neq \alpha \in \pi_{2 n+2} L^{2 n+1}=\mathbf{Z}_{2}$, define $g^{\alpha}: S^{1} \times L^{2 n+1} \rightarrow L^{2 n+1}$ by the composite

$$
S^{1} \times L^{2 n+1} \rightarrow S^{1} \times L^{2 n+1} \vee S^{2 n+2} \stackrel{N^{N \alpha}}{\rightarrow} L^{2 n+1}
$$

where $S^{1} \times L^{2 n+1} \rightarrow S^{1} \times L^{2 n+1} \vee S^{2 n+2}$ is the obvious pinching map. For $[\bar{g}] \in \pi_{1}\left(\operatorname{Aut}\left(L^{2 n+1}, *\right)\right)$, it is easy to see that $\left[g^{\alpha}\right]=[g]+l[h]$, where $g: S^{1} \times L^{2 n+1} \rightarrow L^{2 n+1}$ is induced from $\bar{g}$, and hence $\left[g^{\alpha}\right] \neq[g]$. So, the action of $H^{2 n+2}\left(S^{1} \times L^{2 n+1} ; \mathbf{Z}_{2}\right)=\mathbf{Z}_{2}$ on the liftings of $f$ to $E_{2}$ is effective, i.e., it is an action of $H^{2 n+2}\left(S^{1} \times L^{2 n+1} ; \mathbf{Z}_{2}\right)$ itself rather than a quotient of it.

On the other hand, since $\operatorname{dim}\left(S^{1} \times L^{2 n+1}\right)=2 n+2$, the number of possible liftings of $f$ to $E_{2}$ is the order of the group $\pi_{1}(\operatorname{Aut}(L, *))$. We just prove that it is of order $2 l$. In fact, it is a cyclic group of order $2 l$ generated by the image of $[h] \in \pi_{1}\left(\operatorname{Diff}\left(L^{2 n+1}, *\right)\right)$. This proves the proposition.

Recall that we have defined Aut $M$ as the space of simple homotopy equivalences of $M$. Let $\mathcal{H}(M)$ be the set of all homotopy equivalences. Let $\mathbf{Z}_{l}^{*}$ be the units of $\mathbf{Z}_{l}(l$ odd $)$, and let

$$
\gamma: \pi_{0}\left(\mathcal{H}\left(L^{2 n+1}\right)\right) \rightarrow \operatorname{Aut}\left(\pi_{1} L^{2 n+1}\right)=\mathbf{Z}_{l}^{*}
$$

be defined by $[f] \rightarrow f_{*}: \pi_{1} L^{2 n+1} \rightarrow \pi_{1} L^{2 n+1}$. Then, we have the following result [9].

Proposition 3.2. $\gamma$ is injective and the image is the set of units $k \in \mathbf{Z}_{l}^{*}$ such that $k^{n} \equiv \pm 1 \bmod l$. (The + ones correspond to the orientation-preserving homotopy equivalences.)

Let us now write $L^{2 n+1}=L^{2 n+1}\left(r_{0}, \ldots, r_{n}\right)=S^{2 n+1} / \mathbf{Z}_{l}$ where $\mathbf{Z}_{l}$ acts on $S^{2 n+1}$ by $\tau\left(z_{0}, \ldots, z_{n}\right)=\left(e^{2 \pi i q_{0} / l} z_{0}, \ldots, e^{2 \pi i q_{n} / l} z_{n}\right)$ with $q_{i} r_{i} \equiv 1 \bmod l$. This gives $L^{2 n+1}$ a preferred orientation and a generator $t$ of $\pi_{1} L^{2 n+1}=\mathbf{Z}_{l}$, defined by $t(\theta)=\left(e^{2 \pi \imath \theta q_{0} / l}, 0, \ldots, 0\right)$ for $\theta \in[0,1]$. The diffeomorphism $L\left(r_{0}, \ldots, r_{n}\right) \approx L\left(r_{\sigma(0)}, \ldots, r_{\sigma(n)}\right)$ preserves orientation and the generator for $\sigma$ a permutation of $(0,1, \ldots, n)$; and the diffeomorphism $L\left(r_{0}, \ldots, r_{n}\right)$ $\approx L\left(l-r_{0}, r_{1}, \ldots, r_{n}\right)$ preserves the generator but reserves the orientation. Also if $k \in \mathbf{Z}_{l}^{*}$, there is a diffeomorphism $L\left(r_{0} k, \ldots, r_{n} k\right) \approx L\left(r_{0}, \ldots, r_{n}\right)$.

Following [9], we now define $\Delta(L)=\pi\left(t^{r_{l}}-1\right) \in Q\left[\mathbf{Z}_{l}\right]\left(\mathbf{Z}_{l}\right.$ is now written multiplicatively). Then, if a homotopy equivalence $f$ is simple, we must have $f_{*} \Delta(L)= \pm t^{u} \Delta(L)$ for some $u$. On the other hand, using the diffeomorphisms above and Franz's theorem [4], [9], we have the following proposition. 
Proposition 3.3. If $f$ is a homotopy equivalence such that $f_{*} \Delta(L)=$ $\pm t^{u} \Delta(L), f$ can be represented by a diffeomorphism. Hence, the image of

$$
\pi_{0}\left(\text { Diff } L^{2 n+1}\right) \rightarrow \pi_{0}\left(\mathcal{H}\left(L^{2 n+1}\right)\right)
$$

is exactly the set of homotopy classes of simple homotopy equivalences, i.e.,

$$
\pi_{0}\left(\text { Diff } L^{2 n+1}\right) \rightarrow \pi_{0}\left(\text { Aut } L^{2 n+1}\right) \rightarrow 0 .
$$

Since the map is factored through $\pi_{0}\left(\right.$ Homeo $\left.L^{2 n+1}\right)$, we also have

$$
\pi_{0}\left(\text { Homeo } L^{2 n+1}\right) \rightarrow \pi_{0}\left(\text { Aut } L^{2 n+1}\right) \rightarrow 0 .
$$

(Note that $S=\pi_{0}\left(\right.$ Aut $\left.L^{2 n+1}\right)$.)

IV. Proofs of Theorem 1.1 and Theorem 2.1. In this section, $L^{2 n+1}$ always denotes the lens space $L^{2 n+1}\left(q_{0}, \ldots, q_{n}\right)=S^{2 n+1} / \mathbf{Z}_{l}$ with $\mathbf{Z}_{l} \subset$ $U(n+1)$ generated by

$$
\left(\begin{array}{ccc}
e^{2 \pi l q_{0} / l} & & \\
& \ddots & \\
& & e^{2 \pi l q_{n} / l}
\end{array}\right)
$$

We also assume that $\pi_{1} L^{2 n+1}=\mathbf{Z}_{l}$ is of odd order. Let us now begin with the following lemma.

LEMma 4.1. The maps

$$
\begin{gathered}
{\left[L^{2 n+1} \times D^{2} / L^{2 n+1} \times S^{1} ; G / 0\right] \rightarrow \pi_{0}\left(\bigodot\left(L^{2 n+1}\right)\right),} \\
{\left[L^{2 n+1} \times D^{2} / L^{2 n+1} \times S^{1} ; G / \mathrm{Top}\right] \rightarrow \pi_{0}\left(\bigodot\left(L^{2 n+1}\right)\right)=\pi_{0}\left(\bigodot^{\mathrm{Top}}\left(L^{2 n+1}\right)\right)}
\end{gathered}
$$

are trivial.

Proof. It follows from $\S 3$ that the map

$$
\left[L^{2 n+1} \times D^{2} / L^{2 n+1} \times S^{1} ; G / 0\right] \rightarrow \pi_{0}(\bigodot(L))
$$

is actually factored through

$$
\left[L^{2 n+1} \times D^{2} / L^{2 n+1} \times S^{1}, T / \text { Top }\right] \rightarrow \pi_{0}\left(\bigodot^{\text {Top }}(L)\right)=\pi_{0} \varrho(L),
$$

so it suffices to prove the latter is trivial.

$\left[L^{2 n+1} \times D^{2} / L^{2 n+1} \times S^{1} ; G /\right.$ Top] is always finite, and we shall discuss the 2-torsion part and the odd primary part separately.

(1) The 2-torsion part. The 2-torsion of $\left[L^{2 n+1} \times D^{2} / L^{2 n+1} \times S^{1}\right.$; $G /$ Top] is exactly equal to $Z_{2}$ generated by the composite 


$$
\left[L^{2 n+1} \times D^{2} / L^{2 n+1} \times S^{1}\right]^{\text {proj }} \rightarrow\left(D^{2} / S^{1}\right) \stackrel{K}{\rightarrow} G / \text { Top }
$$

where $K$ generates $\pi_{2}(G /$ Top $)=\mathbf{Z}_{2}$. In terms of normal maps, this is

$$
\text { id } \times(f, b): L^{2 n+1} \times \overline{\left(T^{2}-D^{2}\right)} \rightarrow L^{2 n+1} \times D^{2}
$$

where $(f, b): \overline{T^{2}-D^{2}} \rightarrow D^{2}$ has Kervaire invariant 1 .

By the exactness of the sequence

$$
\begin{aligned}
\pi_{1} \mathscr{F}^{\operatorname{Top}}\left(L^{2 n+1}\right) & =\pi_{2}\left(\text { Aut } L^{2 n+1} ; \text { Homeo } L^{2 n+1}\right) \\
& \rightarrow\left[L^{2 n+1} \times D^{2} / L^{2 n+1} \times S^{1} ; G / \text { Top }\right] \rightarrow \pi_{0}(\mathcal{C}(L))
\end{aligned}
$$

it suffices to prove that this element can be represented by a fiber-preserving homotopy equivalence $h$ of $L^{2 n+1} \times D^{2}$, which is a homeomorphism on $L^{2 n+1} \times S^{1}$. In fact, the map which we shall construct is the identity on the boundary. (So, it comes from $\pi_{2}\left(\right.$ Aut $\left.L^{2 n+1}\right)$.)

Let $L^{2 n+1} \times D^{2} \rightarrow L^{2 n+1}$ be the projection, and change it on a disc in $\operatorname{Int}\left(L^{2 n+1} \times D^{2}\right)$ by a generator of $\pi_{2 n+3}\left(L^{2 n+1}\right)=\mathbf{Z}_{2}$. This obviously gives us an $h$ of the right type, and we only have to prove that it represents the non-zero element in $\left[L^{2 n+1} \times D^{2} / L^{2 n+1} \times S^{1} ; G / \mathrm{Top}\right]_{(2)}$. But this element is recognized by looking at the inverse image of $D^{2}=* \times D^{2} \subset$ $L^{2 n+1} \times D^{2}$ and study the induced framing. In our case, it is $l$ times the generator which is non-trivial since $l$ is odd.

(2) The odd primary part. Here, we use an inductive argument similar to that of [1]. Let $g:(M, \partial M) \rightarrow\left(L^{2 n+1} \times D^{2}, L^{2 n+1} \times S^{1}\right)$ be a normal map, homeomorphic on the boundary. In $L^{2 n+1}$, we have a lens space $L^{2 n-1}$ of codim 2 and we let $f:(N, \partial N) \rightarrow\left(L^{2 n-1} \times D^{2}, L^{2 n-1} \times S^{1}\right)$ be the induced normal map. Suppose by induction that this map is zero in $\pi_{0} e^{T o p}\left(L^{2 n-1}\right)$, i.e., there is a diagram ${ }^{1}$

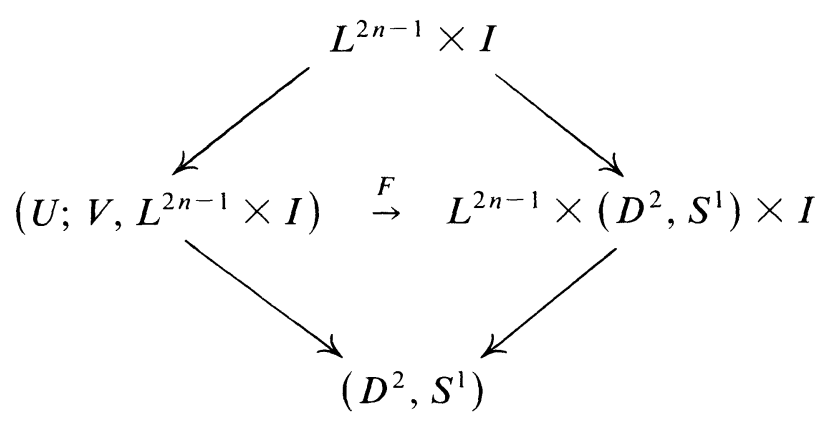

${ }^{1}$ See [8, pp. 420-421]. 
where $F$ is a degree 1 map, $V \rightarrow L^{2 n-1} \times S^{1} \times I$ is a simple homotopy equivalence, $V \rightarrow S^{1} \times I$ is a fibration and $F$ restricts to $f$ on one end while a homeomorphism on the other.

Now, we can extend this cobordism to a cobordism of a neighborhood of $L^{2 n-1} \times D^{2}$ in $L^{2 n+1} \times D^{2}$ with the same properties. By the usual argument of normal cobordism extension with an obvious modification, we may assume that $g$ is a diffeomorphism in a neighborhood of $L^{2 n-1} \times D^{2}$. The complement of a neighborhood of $L^{2 n-1} \times D^{2}$ in $L^{2 n+1}$ $\times D^{2}$ is $S^{1} \times D^{2 n+2}$, so our element lies in the image of

$$
\pi_{0}\left(\bigodot^{\mathrm{Top}}\left(S^{1} \times D^{2 n} ; \text { rel } \partial\right)\right) \rightarrow \pi_{0}\left(\bigodot^{\mathrm{Top}}\left(L^{2 n+1}\right)\right)
$$

'induces' by the inclusion $S^{1} \times D^{2 n} \subset L^{2 n+1}$. But, modulo 2-torsion ${ }^{2}$ which includes the elements from $\mathrm{Wh}_{1}^{+}$, these groups are isomorphic to $L_{2 n+3}^{\text {st }}(\mathbf{Z})$ and $L_{2 n+3}^{\text {st }}\left(\mathbf{Z}_{l}\right)$ of [8] respectively, and the homomorphism is induced by the reduction $\mathbf{Z} \rightarrow \mathbf{Z}_{l}$.

If we can get our induction started, this lemma follows from the following sublemma.

SUBLEMMa 4.2. The homomorphism

$$
L_{i}^{\mathrm{st}}(\mathbf{Z})\left[\frac{1}{2}\right] \rightarrow L_{i}^{\mathrm{st}}(\mathbf{Z})\left[\frac{1}{2}\right]
$$

is trivial for all odd $i$.

Proof of the sublemma. From the exactness of

$$
\begin{gathered}
0 \rightarrow \mathrm{Wh}_{2}(G) /\left\{x-\bar{x} \mid x \in \mathrm{Wh}_{2}(G)\right\} \otimes \mathbf{Z}\left[\frac{1}{2}\right] \rightarrow \\
L_{l}^{\mathrm{st}}(G) \otimes \mathbf{Z}\left[\frac{1}{2}\right] \rightarrow L_{i+2}(G) \otimes \mathbf{Z}\left[\frac{1}{2}\right] \rightarrow 0,
\end{gathered}
$$

we get

$$
\begin{aligned}
& L_{i}^{\text {st }}(\mathbf{Z}) \otimes \mathbf{Z}\left[\frac{1}{2}\right] \simeq L_{i+2}(\mathbf{Z}) \otimes \mathbf{Z}\left[\frac{1}{2}\right] \\
& L_{i}^{\text {st }}\left(\mathbf{Z}_{l}\right) \otimes \mathbf{Z}\left[\frac{1}{2}\right] \simeq \mathrm{Wh}_{2}\left(\mathbf{Z}_{l}\right) /\left\{x-\bar{x} \mid x \in \mathrm{Wh}_{2}\left(\mathbf{Z}_{l}\right)\right\} \otimes \mathbf{Z}\left[\frac{1}{2}\right]
\end{aligned}
$$

\footnotetext{
${ }^{2}$ Igusa recently pointed out that the $\mathrm{Wh}_{1}^{+}\left(\pi_{1} M ; \mathbf{Z}_{2}\right)$ component of the description of $\pi_{0}(\mathcal{P}(M))$ of [7] may have to be modified. So, our description of the subgroup of $\pi_{0}(\mathcal{C}(M))$ coming from $\mathrm{Wh}_{1}^{+}\left(\pi_{1} M ; \mathbf{Z}_{2}\right)$ may also be changed accordingly. But there is no effect on the odd components of $\pi_{0}\left(e\left(L^{2 n+1}\right)\right)$ which is given by $L_{2 n+3}^{\text {st }}\left(\mathbf{Z}_{l}\right)$.
} 
Taking product of the geometric problem with $S^{2}$, we have the following commutative diagram

$$
\begin{aligned}
& L_{l+2}(\mathbf{Z}) \otimes \mathbf{Z}\left[\frac{1}{2}\right] \stackrel{\simeq}{\leftarrow} L_{l}^{\mathrm{st}}(\mathbf{Z}) \otimes Z\left[\frac{1}{2}\right] \quad \rightarrow \quad L_{i}^{\mathrm{st}}\left(\mathbf{Z}_{l}\right) \otimes \mathbf{Z}\left[\frac{1}{2}\right] \\
& L_{i+4}(\mathbf{Z}) \otimes Z\left[\frac{1}{2}\right] \stackrel{\simeq}{\leftarrow} L_{i+2}^{\mathrm{st}}(\mathbf{Z}) \otimes Z\left[\frac{1}{2}\right] \quad \rightarrow \quad L_{i+2}^{\mathrm{st}}\left(\mathbf{Z}_{l}\right) \otimes \mathbf{Z}\left[\frac{1}{2}\right] \\
& \rightarrow \quad \mathrm{Wh}_{2}\left(\mathbf{Z}_{l}\right) /\left\{x-\bar{x} \mid x \in \mathrm{Wh}_{2}\left(Z_{l}\right)\right\} \otimes \mathbf{Z}\left[\frac{1}{2}\right] \\
& \downarrow \simeq \\
& \rightarrow \quad \mathrm{Wh}_{2}\left(\mathbf{Z}_{l}\right) /\left\{x-\bar{x} \mid x \in \mathrm{Wh}_{2}\left(Z_{l}\right)\right\} \otimes \mathbf{Z}\left[\frac{1}{2}\right] .
\end{aligned}
$$

The sublemma follows immediately.

Let us now continue our proof of Lemma 4.1. It remains to show how the induction gets started.

Let us consider the following commutative diagram localized away from 2:

$$
\begin{aligned}
& {\left[L^{5} \times D^{2} / L^{5} \times S^{1} ; G / p L\right] \rightarrow\left[L^{3} \times D^{2} / L^{3} \times S^{1} ; G / \text { Top }\right] \leftarrow\left[S^{1} \times D^{4} / S^{1} \times S^{3} ; G / \text { Top }\right]} \\
& \downarrow \alpha \\
& \pi_{0}\left(e^{\text {Top }}\left(L^{5}\right)\right) \\
& \pi_{0}\left(\bigodot^{\text {Top }}\left(L^{3}\right)\right) \\
& \pi_{0}\left(e^{\mathrm{Top}_{\mathrm{op}}}\left(S^{1} \times D^{2}\right)\right)
\end{aligned}
$$

where $\alpha$ 's are the maps of (7). For $x \in\left[L^{5} \times D^{2} / L^{5} \times S^{1} ; G /\right.$ Top], we see that $\alpha\left(x \mid L^{3}\right)$ is already in the imager of $\pi_{0} e\left(S^{1} \times D^{2}\right.$; rel $\left.\partial\right)$, but the algebraic identification of $\pi_{0}\left(e^{\mathrm{Top}}(M)\right)$ is no longer valid and we can not apply the algebraic sublemma above directly. However, we can get around this by multiplying everything with simply connected manifold $N$ of Euler characteristic 1 or $2^{i}$. Observe that $\alpha(x)=0$ for $x \in\left[L^{5} \times D^{2} / L^{5} \times S^{1}\right.$; $G /$ Top] if and only if $\alpha(x \times N)=0$, since the product formula is valid for $L^{5}$. So, we can go through the same argument with this problem, noting that $x \times N \mid L^{3} \times N$ still lies in the image of $\left[S^{1} \times D^{4} \times N / S^{1} \times\right.$ $S^{3} \times N ; G /$ Top]. Hence, $\alpha\left(x \times N \mid L^{3} \times N\right)$ lies in the image of $\pi_{0}\left(\bigodot^{\mathrm{Top}}\left(S^{1} \times D^{2} \times N\right.\right.$; rel $\left.\left.\partial\right)\right)$. Now, we can use the sublemma and get $\alpha\left(x \times N \mid L^{3} \times N\right)=0$. Therefore, $\alpha(x \times N)$ lies in the image of $\pi_{0}\left(\bigodot^{\text {Top }}\left(S^{1} \times D^{4} \times N\right.\right.$; rel $\left.\left.\partial\right)\right)$ and it is zero by the same sublemma. This completes the proof of the lemma.

LeMma 4.3. $\pi_{0}\left(\mathscr{F}\left(L^{2 n+1}\right)\right)=\pi_{0}\left(\mathcal{C}\left(L^{2 n+1}\right)\right) \oplus H$ and

$$
\pi_{0}\left(\mathscr{F}^{\mathrm{Top}}\left(L^{2 n+1}\right)\right)=\pi_{0}\left(\mathcal{C}\left(L^{2 n+1}\right)\right) .
$$


Proof. Consider the map of (6) to (7) and we have the following commutative diagram

$$
\begin{aligned}
& \rightarrow \pi_{0}\left(e\left(L^{2 n+1}\right)\right) \rightarrow \pi_{0}\left(\mathscr{F}\left(L^{2 n+1}\right)\right) \rightarrow\left[L^{2 n+1} \times I / L^{2 n+1} \times \partial I ; G / 0\right] \stackrel{\theta_{2}}{\rightarrow} \quad L_{2 n+2}\left(Z_{l}\right) \\
& \downarrow \simeq \quad \downarrow \quad \downarrow \quad \downarrow \simeq \\
& \rightarrow \pi_{0}\left(e^{\mathrm{Top}}\left(L^{2 n+1}\right)\right) \rightarrow \pi_{0}\left(\mathscr{F}^{\mathrm{Top}}\left(L^{2 n+1}\right)\right) \rightarrow\left[L^{2 n+1} \times I / L^{2 n+1} \times \partial I ; G / \mathrm{Top}\right] \stackrel{\theta_{2}}{\rightarrow} L_{2 n+2}\left(Z_{l}\right) \\
& {\left[D^{2 n+2} / \partial D^{2 n+2} ; G / \text { Top }\right] \quad \stackrel{\theta_{3}}{\rightarrow} \quad L_{2 n+2}(1)}
\end{aligned}
$$

where $\theta_{1}, \theta_{2}, \theta_{3}$ are the surgery maps.

It follows from Lemma 4.1 that

$$
\begin{gathered}
\pi_{0}\left(\mathscr{F}^{\text {Top }}\left(L^{2 n+1}\right)\right)=\pi_{0}\left(\bigodot\left(L^{2 n+1}\right)\right), \\
\pi_{0}\left(\mathscr{F}\left(L^{2 n+1}\right)\right)=\pi_{0}\left(\bigodot\left(L^{2 n+1}\right)\right) \oplus H^{\prime}
\end{gathered}
$$

where $H^{\prime}$ is the image of $\pi_{0}\left(\mathscr{F}\left(L^{2 n+1}\right)\right)$ in $\left[L^{2 n+1} \times I / L^{2 n+1} \times \partial I ; G / 0\right]$.

Consider the following commutative diagram

$$
\begin{array}{ccccc}
\pi_{0}\left(\mathscr{F}\left(L^{2 n+1}\right)\right) & \rightarrow & {\left[L^{2 n+1} \times I / L^{2 n+1} \times \partial I \text {; Top } / 0\right]} & & \\
\downarrow & & & & \\
\pi_{0}\left(S\left(L^{2 n+1} \times I ; \text { rel } \partial\right)\right) & \rightarrow & {\left[L^{2 n+1} \times I \times L^{2 n+1} \times \partial I ; G / 0\right]} & \stackrel{\theta_{1}}{\rightarrow} & L_{2 n+2}\left(\mathbf{Z}_{l}\right) \\
\downarrow & & & \downarrow \simeq \\
\pi_{0}\left(S^{\text {Top }}\left(L^{2 n+1} \times I \text {; rel } \partial\right)\right) & \rightarrow & {\left[K^{2 n+1} \times I / L^{2 n+1} \times \partial I ; G / \text { Top }\right]} & \stackrel{\theta_{2}}{\rightarrow} & L_{2 n+2}\left(\mathbf{Z}_{l}\right) .
\end{array}
$$

The injectivity of $\theta_{2}$ implies that

$$
\begin{aligned}
\pi_{0}\left(\mathscr{F}\left(L^{2 n+1}\right)\right) & \rightarrow\left[L^{2 n+1} \times I / L^{2 n+1} \times \partial I ; G / 0\right] \\
& \rightarrow\left[L^{2 n+1} \times I / L^{2 n+1} \times \partial I ; G / \text { Top }\right]
\end{aligned}
$$

is trivial and $H^{\prime}$ is contained in $H$, the image of $\left[L^{2 n+1} \times I / L^{2 n+1} \times \partial I\right.$; Top/0] in $\left[L^{2 n+1} \times I / L^{2 n+1} \times \partial I ; G / 0\right]$. On the other hand, it is obvious that $H \subset H^{\prime}$ and the lemma is proved.

Proofs of Theorem 1.1 and Theorem 2.1. Let us look at the exact sequence

$$
\begin{aligned}
& \rightarrow \pi_{1}\left(\text { Diff } L^{2 n+1}\right) \rightarrow \pi_{1}\left(\text { Aut } L^{2 n+1}\right) \rightarrow \\
& \pi_{0}\left(\mathscr{F}\left(L^{2 n+1}\right)\right) \rightarrow \pi_{0}\left(\text { Diff } L^{2 n+1}\right) \rightarrow \pi_{0}\left(\text { Aut } L^{2 n+1}\right) .
\end{aligned}
$$

By Propositions 3.1 and 3.2 this reduces to

$$
\begin{aligned}
0 & \rightarrow \pi_{0}\left(\mathscr{F}\left(L^{2 n+1}\right)\right) \rightarrow \pi_{0}\left(\text { Diff } L^{2 n+1}\right) \\
& \rightarrow \pi_{0}\left(\text { Aut } L^{2 n+1}\right)=S \rightarrow 0 .
\end{aligned}
$$


Similarly, we have

$$
\begin{aligned}
0 & \rightarrow \pi_{0}\left(\mathscr{F}^{\operatorname{Top}}\left(L^{2 n+1}\right)\right) \rightarrow \pi_{0}\left(\text { Homeo } L^{2 n+1}\right) \\
& \rightarrow S \rightarrow 0 .
\end{aligned}
$$

The theorems follow from Lemma 4.3.

\section{REFERENCES}

[1] W. Browder, Free $Z_{p}$-actions on Homotopy Spheres, Topology of Manifolds Markham, 1970, 217-226.

[2] D. Burghelea, R. Lashof and M. Rothenberg, Groups of Automorphisms of Manifolds, Lecture Notes in Math., 473 Springer-Verlag, (1975).

[3] J. Cerf, La stratification naturelle des espaces de fonctions différentiables réelles et le théorème de la pseudo-isotopie, Pub. Math. I.H.E.S., 39 (1970), 5-173.

[4] W. Franz, Über die Torsion einer Überdeckung, T. Reine Angew. Math., 173 (1935), 245-254.

[5] A. Hatcher, Higher simple homotopy theory, Ann. of Math., 102 (1975), 101-137.

[6] Concordance spaces, higher simple homotopy theory and applications, Proc. Symp. Pure Math., 82 (1978).

[7] A. Hatcher and J. Wagoner, Pseudo-isotopies of compact manifolds, Astérisque, 6 (1973), Soc. de France.

[8] W. C. Hsiang and R. W. Sharpe, Parametrized surgery and isotopy, Pacific J. Math., 67 (1976), 401-459.

[9] J. Milnor, Whitehead torsion, Bull. Amer. Math. Soc., 72 (1966), 358-426.

[10] E. K. Pederson, Topological concordances, Inv. Math., 38 (1977), 255-267.

[11] E. P. Peterson and N. Stein, Secondary cohomology operations: two formulas, Amer. J. Math., 81 (1959), 281-305.

[12] F. S. Quinn, A geometric formulation of surgery, Ph.D. thesis, Princeton University, 1969.

[13] J. L. Shaneson, Wall's surgery obstruction groups for $G \times Z$, Ann. of Math., 90 (1969), 296-334.

[14] E. Spanier, Algebraic Topology, McGraw-Hill, 1966.

Received February 26, 1981. The first author was partially supported by an NSF Grant.

PRINCETON UNIVERSITY

PRINCETON, NJ 08544 



\title{
PACIFIC JOURNAL OF MATHEMATICS EDITORS
}

\author{
Donald BabBitT (Managing Editor) \\ University of California \\ Los Angeles, CA 90024 \\ Hugo RossI \\ University of Utah \\ Salt Lake City, UT 84112 \\ C. C. Moore and Arthur Ogus \\ University of California \\ Berkeley, CA 94720
}

J. DugundiI

Department of Mathematics

University of Southern California

Los Angeles, CA 90089-1113

R. FinN and H. SAMELSON

Stanford University

Stanford, CA 94305

\section{ASSOCIATE EDITORS}
R. ARENS
E. F. BECKENBACH
B. H. NeUmanN
F. WOLF
K. YosHida
(1906-1982)

\section{SUPPORTING INSTITUTIONS}

\author{
UNIVERSITY OF ARIZONA \\ UNIVERSITY OF BRITISH COLUMBIA \\ CALIFORNIA INSTITUTE OF TECHNOLOGY \\ UNIVERSITY OF CALIFORNIA \\ MONTANA STATE UNIVERSITY \\ UNIVERSITY OF NEVADA, RENO \\ NEW MEXICO STATE UNIVERSITY \\ OREGON STATE UNIVERSITY
}

\author{
UNIVERSITY OF OREGON \\ UNIVERSITY OF SOUTHERN CALIFORNIA \\ STANFORD UNIVERSITY \\ UNIVERSITY OF HAWAII \\ UNIVERSITY OF TOKYO \\ UNIVERSITY OF UTAH \\ WASHINGTON STATE UNIVERSITY \\ UNIVERSITY OF WASHINGTON
}

The Supporting Institutions listed above contribute to the cost of publication of this Journal, but they are not owners or publishers and have no responsibility for its content or policies.

Mathematical papers intended for publication in the Pacific Journal of Mathematics should be in typed form or offset-reproduced (not dittoed), double spaced with large margins. Please do not use built up fractions in the text of the manuscript. However, you may use them in the displayed equations. Underline Greek letters in red, German in green, and script in blue. The first paragraph must be capable of being used separately as a synopsis of the entire paper. In particular it should contain no bibliographic references. Please propose a heading for the odd numbered pages of less than 35 characters. Manuscripts, in triplicate, may be sent to any one of the editors. Please classify according to the scheme of Math. Reviews, Index to Vol. 39. Supply name and address of author to whom proofs should be sent. All other communications should be addressed to the managing editor, or Elaine Barth, University of California, Los Angeles, California 90024.

There are page-charges associated with articles appearing in the Pacific Journal of Mathematics. These charges are expected to be paid by the author's University, Government Agency or Company. If the author or authors do not have access to such Institutional support these charges are waived. Single authors will receive 50 free reprints; joint authors will receive a total of 100 free reprints. Additional copies may be obtained at cost in multiples of 50 .

The Pacific Journal of Mathematics is issued monthly as of January 1966. Regular subscription rate: $\$ 132.00$ a year (6 Vol., 12 issues). Special rate: $\$ 66.00$ a year to individual members of supporting institutions.

Subscriptions, orders for numbers issued in the last three calendar years, and changes of address should be sent to Pacific Journal of Mathematics, P.O. Box 969, Carmel Valley, CA 93924, U.S.A. Old back numbers obtainable from Kraus Periodicals Co., Route 100, Millwood, NY 10546.

The Pacific Journal of Mathematics ISSN $0030-8730$ is published monthly by the Pacific Journal of Mathematics at P.O. Box 969, Carmel Valley, CA 93924. Application to mail at Second-class postage rates is pending at Carmel Valley, California, and additional mailing offices. Postmaster: Send address changes to Pacific Journal of Mathematics, P. O. Box 969, Carmel Valley, CA 93924.

PUBLISHED BY PACIFIC JOURNAL OF MATHEMATICS, A NON-PROFIT CORPORATION

Copyright $\odot 1983$ by Pacific Journal of Mathematics 


\section{Pacific Journal of Mathematics}

Vol. 109, No. $2 \quad$ June, 1983

Tibor Bisztriczky, On the singularities of almost-simple plane curves . . . . 257

Peter B. Borwein, On Sylvester's problem and Haar spaces .............. 275

Emilio Bujalance, Cyclic groups of automorphisms of compact nonorientable Klein surfaces without boundary ............... 279

Robert Jay Daverman and John J. Walsh, Acyclic decompositions of

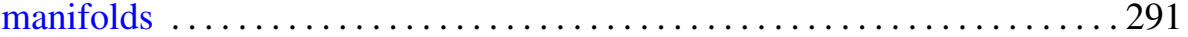

Lester Eli Dubins, Bernstein-like polynomial approximation in higher

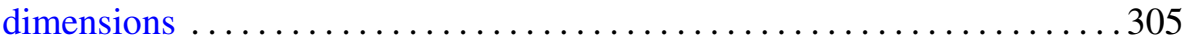

Allan L. Edelson and Jerry Dee Schuur, Nonoscillatory solutions of

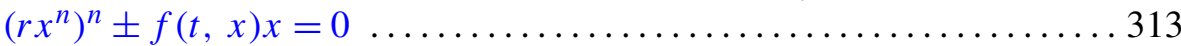

Akira Endô, On units of pure quartic number fields ................. 327

Hector O. Fattorini, A note on fractional derivatives of semigroups and

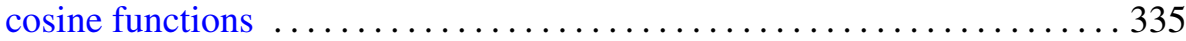

Ronald Fintushel and Peter Sie Pao, Circle actions on homotopy spheres with codimension 4 fixed point set ........................ 349

Stephen Michael Gagola, Jr., Characters vanishing on all but two

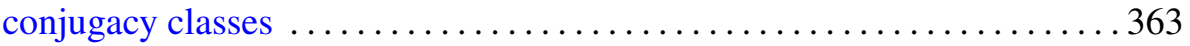

Saverio Giulini, Singular characters and their $L^{p}$ norms on classical Lie

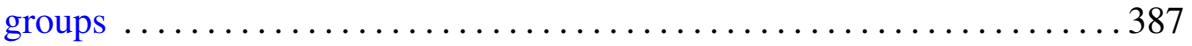

Willy Govaerts, Locally convex spaces of non-Archimedean valued continuous functions

Wu-Chung Hsiang and Bjørn Jahren, A remark on the isotopy classes of diffeomorphisms of lens spaces $\ldots . \ldots \ldots \ldots \ldots \ldots$

Hae Soo Oh, Compact connected Lie groups acting on simply connected 4-manifolds

Frank Okoh and Frank A. Zorzitto, Subsystems of the polynomial system

Knut Øyma, An interpolation theorem for $H_{E}^{\infty}$

Nikolaos S. Papageorgiou, Nonsmooth analysis on partially ordered vector spaces. II. Nonconvex case, Clarke's theory ........ 